\title{
Coupled Sine-Gordon Systems in DNA Dynamics
}

\author{
Liya Liu' ${ }^{1}$ and Chuanzhong $L i \mathbb{D}^{2}$ \\ ${ }^{1}$ Department of Preventive Medicine, School of Medicine, Ningbo University, Ningbo 315211, China \\ ${ }^{2}$ Department of Mathematics, Ningbo University, Ningbo 315211, China
}

Correspondence should be addressed to Chuanzhong Li; lichuanzhong@nbu.edu.cn

Received 14 March 2018; Accepted 16 April 2018; Published 15 May 2018

Academic Editor: Zhijun Qiao

Copyright (C) 2018 Liya Liu and Chuanzhong Li. This is an open access article distributed under the Creative Commons Attribution License, which permits unrestricted use, distribution, and reproduction in any medium, provided the original work is properly cited.

\begin{abstract}
We consider a model called the coupled sine-Gordon equation for DNA dynamics by introducing two double helix structures. The second double helix structure is unilaterally influenced by the first one. The completely integrable coupled sine-Gordon equation admits kink-antikink solitons with increased width representing a wide base pair opening configuration in DNA. Also we propose another coupled sine-Gordon model with variable coefficients for DNA dynamics under an inhomogeneous background. We find that the inhomogeneous DNA model has many interesting localized nonrational rogue wave solutions. We can find that the appearance of the rogue waves (possibly means the genetic mutation) in the nonlinear DNA model is highly related to the inhomogeneity.
\end{abstract}

\section{Introduction}

The sine-Gordon equation is an important integrable equation which describes many interesting phenomena including the DNA double helix molecule [1], dynamics of coupled pendulums, Josephson junction arrays [2], living cellular structures [3], and the crystalline lattices [4]. In the DNA double helix structure, the two parallel chains of nucleotides (strands) connect with dipole-dipole interaction along the strands. The two strands are bound to each other through hydrogen bonds between the complementary bases [5].

Besides in life sciences, the scalar sine-Gordon equation originates in differential geometry and has profound applications in physics which can be seen from a recent review [6]. In [7], the nonrational rogue waves can be induced by the inhomogeneity background of the sine-Gordon equation.

Similarly as the method in our previous work in $[8,9]$, we construct the general $Z_{n}$-sine-Gordon and $Z_{n}$-sinh-Gordon systems which contain many multicomponent sine-Gordon type and sinh-Gordon type equations in [10]. Meanwhile, we give the Bäcklund transformations of the $Z_{n}$-sine-Gordon and the nonlinear superposition formula and Lax pairs.

This paper is arranged as follows. In Section 2, we recall some basic facts about the classical sine-Gordon equation including its Lax pair and Bäcklund transformation. In Section 3, we recall the coupled sine-Gordon equation and its Lax pair. After this, we will construct the Bäcklund transformation of the inhomogeneous coupled sine-Gordon equation and, from the Bäcklund transformation, we derive many solutions including the rogue wave solutions of some special inhomogeneous coupled sine-Gordon equation.

\section{Sine-Gordon Equation}

Under certain circumstances, the DNA can open the hydrogen bonds between conjugated pairs, exposing the unpaired bases to the action of external ligands. To model this motion each base of a strand is assumed to be coupled with the nextneighbour bases of the same strand by the elastic backbone restoring forces and with the complementary base in the opposite strand by the anharmonic potential used to model the hydrogen bond. One take as canonical variables the deflection angles $\phi_{i}$ and $\phi_{i}^{\prime}$ that two complementary bases form with the line passing from the attaching points of the bases to the strands.

In [11], they construct a Hamiltonian of DNA dynamics:

$$
\begin{aligned}
H & =\sum_{n}\left[\frac{I}{2}\left(\dot{\phi}_{n}^{2}+\dot{\phi}_{n}^{\prime 2}\right)+J\left[2-\cos \left(\phi_{n+1}-\phi_{n}\right)\right.\right. \\
& \left.-\cos \left(\phi_{n+1}^{\prime}-\phi_{n}^{\prime}\right)\right]-\eta\left[1-\cos \left(\phi_{n}-\phi_{n}^{\prime}\right)\right]+h\left\{2 q_{0}^{2}\right.
\end{aligned}
$$




$$
\begin{aligned}
& -\left[\sin \left(\phi_{n+1}-\phi_{n}\right)-q_{0}\right]^{2} \\
& \left.\left.-\left[\sin \left(\phi_{n+1}^{\prime}-\phi_{n}^{\prime}\right)-q_{0}\right]^{2}\right\}\right],
\end{aligned}
$$

by introducing the helical structure through twist deformation of double helix structure in analogy with the structure of helimagnet and cholesteric liquid crystal system. Here $I=1 / 2 A^{2}$ is the moment of inertia of the bases around the axes at the $n$th site and $J$ and $A$, respectively, represent the ferromagnetic spin-spin exchange interaction and uniaxial magnetocrystalline anisotropy with the easy axis along $z$ direction. $h$ denotes the elastic constant associated with the twist deformation and $\eta$ is a constant. Having formed the Hamiltonian, the dynamics of the DNA with double helix molecule can be understood by constructing the Hamilton's equations of motion corresponding to the Hamiltonian (1) as

$$
\begin{aligned}
I \phi_{n t t}= & {\left[J+2 h \cos \left(\phi_{n+1}-\phi_{n}\right)\right] \sin \left(\phi_{n+1}-\phi_{n}\right) } \\
& -\left[J+2 h \cos \left(\phi_{n}-\phi_{n-1}\right)\right] \sin \left(\phi_{n}-\phi_{n-1}\right) \\
& +\eta \sin \left(\phi_{n}-\phi_{n}^{\prime}\right) \\
& -2 h q_{0}\left[\cos \left(\phi_{n+1}-\phi_{n}\right)-\cos \left(\phi_{n}-\phi_{n-1}\right)\right], \\
I \phi_{n t t}^{\prime}= & {\left[J+2 h \cos \left(\phi_{n+1}^{\prime}-\phi_{n}^{\prime}\right)\right] \sin \left(\phi_{n+1}^{\prime}-\phi_{n}^{\prime}\right) } \\
& -\left[J+2 h \cos \left(\phi_{n}^{\prime}-\phi_{n-1}^{\prime}\right)\right] \sin \left(\phi_{n}^{\prime}-\phi_{n-1}^{\prime}\right) \\
& +\eta \sin \left(\phi_{n}^{\prime}-\phi_{n}\right) \\
& -2 h q_{0}\left[\cos \left(\phi_{n+1}^{\prime}-\phi_{n}^{\prime}\right)-\cos \left(\phi_{n}^{\prime}-\phi_{n-1}^{\prime}\right)\right] .
\end{aligned}
$$

Equations (2a) and (2b) describe the dynamics of the DNA double helix at the discrete level when the helical nature of the molecule is represented in the form of a twist-like deformation.

Under the approximation by supposing that the difference in angular rotation of bases with respect to neighbouring bases along the two strands is small, the discrete equations of motion after redefinition of the time and the parameter $\eta$ reduce to

$$
\begin{aligned}
& \phi_{t t}=\frac{(J+2 h)}{I} \phi_{z z}+\eta \sin \left(\phi-\phi^{\prime}\right), \\
& \phi_{t t}^{\prime}=\frac{(J+2 h)}{I} \phi_{z z}^{\prime}+\eta \sin \left(\phi^{\prime}-\phi\right),
\end{aligned}
$$

in the continuum limit. After suitable rescaling of the variable $z$, (3a) and (3b) become

$$
\Psi_{t t}-\Psi_{z z}+\sin \Psi=0,
$$

where $\Psi=\phi-\phi^{\prime}$ and we have further chosen $2 \eta=-1$.

By introducing two variables $x=t+z, y=t-z$, the equation can be simplified to the following well-known sineGordon equation:

$$
\Psi_{x y}=\sin \Psi .
$$

2.1. Lax Equations and Bäcklund Transformations. Let us explore the source of the matrix form of the sine-Gordon equation (5). The mathematical method of the soliton equation was derived mainly through zero curvature equation. The zero curvature equation is as follows.

$$
M_{t}-N_{x}+[M, N]=0, \quad[M, N]=M N-N M .
$$

We can export many soliton equations by choosing appropriate values of $M$ and $N$. The sine-Gordon equation has the following form with

$$
\begin{aligned}
& M=\left(\begin{array}{cc}
-i \lambda & q \\
r & i \lambda
\end{array}\right), \\
& N=\left(\begin{array}{cc}
A & B \\
C & -A
\end{array}\right) .
\end{aligned}
$$

Here the zero curvature equation (6) becomes

$$
\begin{aligned}
A_{x} & =q c-r B \\
q_{t} & =B_{x}+2 i \lambda B+2 q A \\
r_{t} & =C_{x}-2 i \lambda C-2 r A .
\end{aligned}
$$

For example, we choose appropriate values of $A, B$, and $C$ as

$$
\begin{aligned}
& A=\frac{a}{\lambda}, \\
& B=\frac{b}{\lambda}, \\
& C=\frac{c}{\lambda},
\end{aligned}
$$

where

$$
\begin{aligned}
& a=\frac{i}{4} \cos u, \\
& b=c=\frac{i}{4} \sin u, \\
& q=r=-\frac{u_{x}}{2} .
\end{aligned}
$$

Then we can get the well-known sine-Gordon equation as follows:

$$
u_{x t}=\sin u \text {. }
$$

$u$ is a solution of (11), and, under the following Bäcklund transformation, $u^{\prime}$ is another solution of (11).

$$
\begin{aligned}
& \left(\frac{u^{\prime}+u}{2}\right)_{x}=a \sin \frac{u^{\prime}-u}{2}, \\
& \left(\frac{u^{\prime}-u}{2}\right)_{t}=\frac{1}{a} \sin \frac{u^{\prime}+u}{2} .
\end{aligned}
$$

\section{Coupled Sine-Gordon Equation}

In this section, we will consider the dynamics of two DNA double helix molecules by constructing the Hamilton's equations of motion as 


$$
\begin{aligned}
& I \phi_{n t t} \\
& =\left[J+2 h \cos \left(\phi_{n+1}-\phi_{n}\right)\right] \sin \left(\phi_{n+1}-\phi_{n}\right) \\
& -\left[J+2 h \cos \left(\phi_{n}-\phi_{n-1}\right)\right] \sin \left(\phi_{n}-\phi_{n-1}\right) \\
& +\eta \sin \left(\phi_{n}-\phi_{n}^{\prime}\right) \\
& -2 h q_{0}\left[\cos \left(\phi_{n+1}-\phi_{n}\right)-\cos \left(\phi_{n}-\phi_{n-1}\right)\right], \\
& I \phi_{n t t}^{\prime} \\
& =\left[J+2 h \cos \left(\phi_{n+1}^{\prime}-\phi_{n}^{\prime}\right)\right] \sin \left(\phi_{n+1}^{\prime}-\phi_{n}^{\prime}\right) \\
& -\left[J+2 h \cos \left(\phi_{n}^{\prime}-\phi_{n-1}^{\prime}\right)\right] \sin \left(\phi_{n}^{\prime}-\phi_{n-1}^{\prime}\right) \\
& +\eta \sin \left(\phi_{n}^{\prime}-\phi_{n}\right) \\
& -2 h q_{0}\left[\cos \left(\phi_{n+1}^{\prime}-\phi_{n}^{\prime}\right)-\cos \left(\phi_{n}^{\prime}-\phi_{n-1}^{\prime}\right)\right] \text {, } \\
& =\left[J+2 h \cos \left(\psi_{n+1}-\psi_{n}\right)\right] \sin \left(\psi_{n+1}-\psi_{n}\right) \\
& -\left[J+2 h \cos \left(\psi_{n}-\psi_{n-1}\right)\right] \sin \left(\psi_{n}-\psi_{n-1}\right) \\
& +\eta \psi_{n} \cos \left(\phi_{n}-\phi_{n}^{\prime}\right) \\
& -2 h q_{0}\left[\cos \left(\psi_{n+1}-\psi_{n}\right)-\cos \left(\psi_{n}-\psi_{n-1}\right)\right], \\
& I \psi_{n t t}^{\prime} \\
& =\left[J+2 h \cos \left(\psi_{n+1}^{\prime}-\psi_{n}^{\prime}\right)\right] \sin \left(\psi_{n+1}^{\prime}-\psi_{n}^{\prime}\right) \\
& -\left[J+2 h \cos \left(\psi_{n}^{\prime}-\psi_{n-1}^{\prime}\right)\right] \sin \left(\psi_{n}^{\prime}-\psi_{n-1}^{\prime}\right) \\
& +\eta \psi_{n}^{\prime} \cos \left(\phi_{n}^{\prime}-\phi_{n}\right) \\
& -2 h q_{0}\left[\cos \left(\psi_{n+1}^{\prime}-\psi_{n}^{\prime}\right)-\cos \left(\psi_{n}^{\prime}-\psi_{n-1}^{\prime}\right)\right] .
\end{aligned}
$$

Equations (13a)-(14b) can possibly describe the dynamics of the interaction of two DNA double helix structures with each other at the discrete level in the form of a twist-like deformation.

Hence, under the small angle approximation, in the continuum limit, that is,

$$
\phi_{i+1}-2 \phi_{i}+\phi_{i-1} \longmapsto \phi_{z z}
$$

the discrete equations of motion (13a)-(14b) after suitable rescaling of time and redefinition of the parameter $\eta$ reduce to

$$
\begin{aligned}
& \phi_{t t}=\frac{(J+2 h)}{I} \phi_{z z}+\eta \sin \left(\phi-\phi^{\prime}\right), \\
& \phi_{t t}^{\prime}=\frac{(J+2 h)}{I} \phi_{z z}^{\prime}+\eta \sin \left(\phi^{\prime}-\phi\right), \\
& \psi_{t t}=\frac{(J+2 h)}{I} \psi_{z z}+2 \eta \psi \cos \left(\phi-\phi^{\prime}\right), \\
& \psi_{t t}^{\prime}=\frac{(J+2 h)}{I} \psi_{z z}^{\prime}+2 \eta \psi^{\prime} \cos \left(\phi^{\prime}-\phi\right) .
\end{aligned}
$$

Adding and subtracting (17a) and (17b) and after suitable rescaling of the variable $z$, we obtain

$$
\begin{array}{r}
\Phi_{t t}-\Phi_{z z}+\sin \Phi=0, \\
\Psi_{t t}-\Psi_{z z}+\Psi \cos \Phi=0,
\end{array}
$$

where $\Phi=\phi-\phi^{\prime}, \Psi=\psi-\psi^{\prime}$, and we have further chosen $\eta=-1$.

3.1. Lax Equations of the Coupled Sine-Gordon Equation. In this section, we will consider the following coupled sineGordon equation:

$$
\begin{aligned}
& u_{x t}=\sin u, \\
& v_{x t}=v \cos u .
\end{aligned}
$$

The Lax equation of the coupled sine-Gordon equation is as follows [10]:

$$
\begin{aligned}
& \phi_{x}=M \phi, \\
& \phi_{t}=N \phi ;
\end{aligned}
$$

when

$$
\begin{aligned}
& M=\left(\begin{array}{cc}
-i \lambda & q \\
r & i \lambda
\end{array}\right)=\left(\begin{array}{cccc}
-i \lambda & 0 & -\frac{1}{2} u_{x} & 0 \\
0 & -i \lambda & -\frac{1}{2} u_{1 x} & -\frac{1}{2} u_{x} \\
\frac{1}{2} u_{x} & 0 & i \lambda & 0 \\
\frac{1}{2} u_{1 x} & \frac{1}{2} u_{x} & 0 & i \lambda
\end{array}\right), \\
& N=\left(\begin{array}{cc}
A & B \\
C & -A
\end{array}\right) \\
& =\left(\begin{array}{cccc}
\frac{i}{4 \lambda} \cos u & 0 & \frac{i}{4 \lambda} \sin u & 0 \\
-\frac{i}{4 \lambda} v \sin u & \frac{i}{4 \lambda} \cos u & \frac{i}{4 \lambda} v \cos u & \frac{i}{4 \lambda} \cos u \\
\frac{i}{4 \lambda} \sin u & 0 & -\frac{i}{4 \lambda} \cos u & 0 \\
\frac{i}{4 \lambda} v \cos u & \frac{i}{4 \lambda} \sin u & \frac{i}{4 \lambda} v \sin u & -\frac{i}{4 \lambda} \cos u
\end{array}\right),
\end{aligned}
$$

we can export the Lax equation of the coupled sine-Gordon equation.

\section{Inhomogeneous Coupled Sine-Gordon Equation}

The inhomogeneous system is modeled by the following coupled sine-Gordon equation with variable coefficients:

$$
\begin{aligned}
& U_{x t}=m(x, t) \sin U, \\
& V_{x t}=m(x, t) V \cos U .
\end{aligned}
$$

Here $m(x, t)$ represents the inhomogeneity of the system. If $m(x, t)=X_{x}(x) T_{t}(t)$, then the following transformation: 

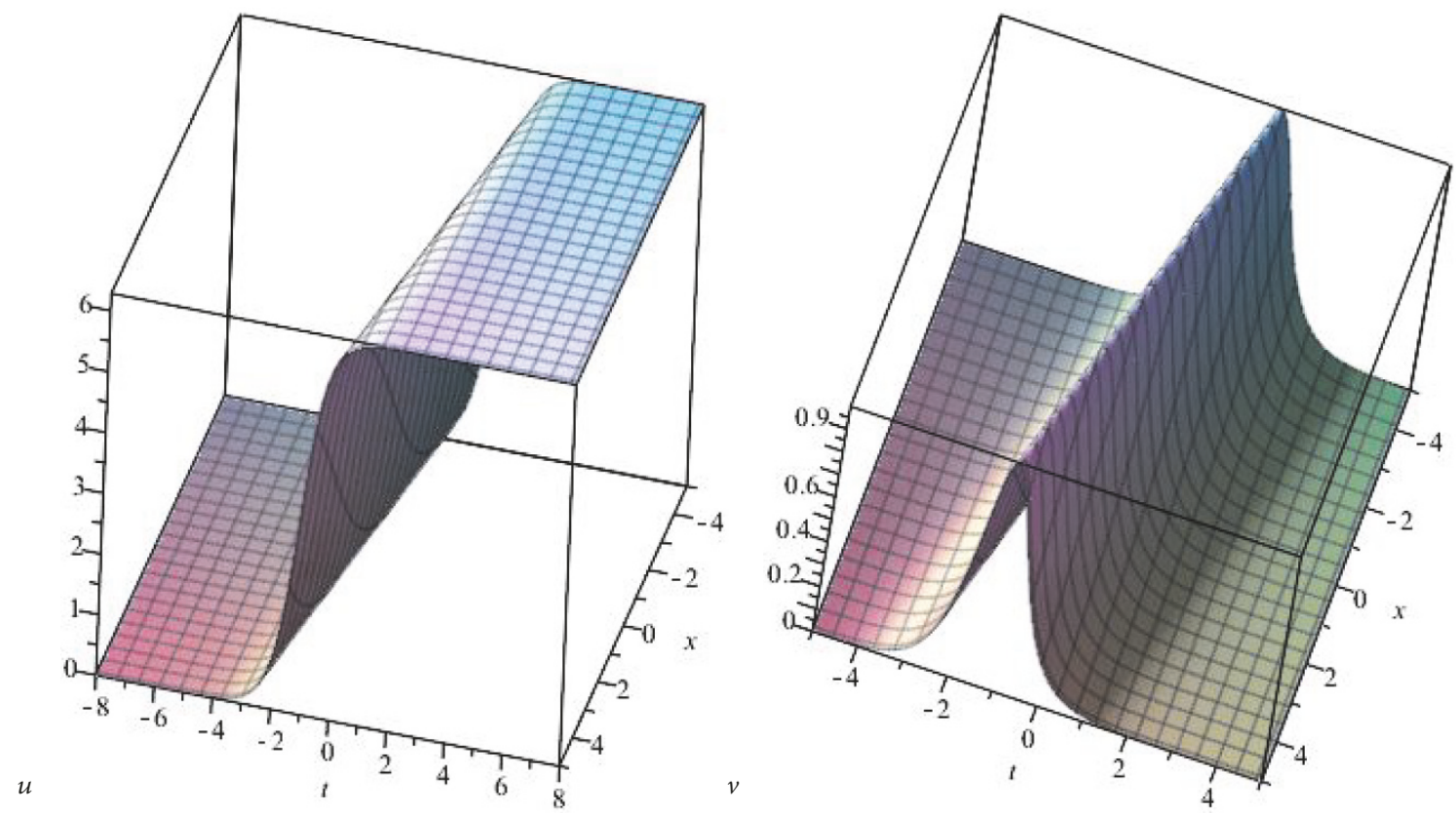

FIGURE 1: The solution $\left(u_{1}, v_{1}\right)$ of the coupled sine-Gordon equation when $a=0.5$.

$U(x, t)=u(X(x), T(t))$, will map the inhomogeneous coupled sine-Gordon equation to the following standard coupled sine-Gordon equation:

$$
\begin{aligned}
& \frac{\partial^{2} u(X, T)}{\partial X \partial T}=\sin u, \\
& \frac{\partial^{2} v(X, T)}{\partial X \partial T}=v \cos u,
\end{aligned}
$$

which has the following Bäcklund transformation:

$$
\begin{aligned}
& \left(\frac{u^{\prime}+u}{2}\right)_{X}=a \sin \frac{u^{\prime}-u}{2}, \\
& \left(\frac{v^{\prime}+v}{2}\right)_{X}=\frac{v^{\prime}-v}{2} \cos \frac{u^{\prime}-u}{2}, \\
& \left(\frac{u^{\prime}-u}{2}\right)_{T}=\frac{1}{a} \sin \frac{u^{\prime}+u}{2}, \\
& \left(\frac{v^{\prime}-v}{2}\right)_{T}=\frac{1}{a} \frac{v+v}{2} \cos \frac{u^{\prime}+u}{2} .
\end{aligned}
$$

There is no doubt that it is also the Bäcklund transformation of the coupled sine-Gordon equation when $X=x, T=t$.

Letting $u=v=0$, then the first equation of the Bäcklund transformation is as follows:

$$
\begin{aligned}
& \left(\frac{u^{\prime}}{2}\right)_{X}=a \sin \frac{u^{\prime}}{2} \\
& \left(\frac{u^{\prime}}{2}\right)_{T}=\frac{1}{a} \sin \frac{u^{\prime}}{2}
\end{aligned}
$$

and the second sets of equation is as follows:

$$
\begin{aligned}
& \left(\frac{v^{\prime}}{2}\right)_{X}=a \frac{v^{\prime}}{2} \cos \frac{u^{\prime}}{2} \\
& \left(\frac{v^{\prime}}{2}\right)_{T}=\frac{1}{a} \frac{v^{\prime}}{2} \cos \frac{u^{\prime}}{2} .
\end{aligned}
$$

Then we derive the following new solution:

$$
\begin{aligned}
& u=4 \arctan e^{a X+a^{-1} T} \\
& v=2 \frac{e^{\left(a X+a^{-1} T\right)}}{e^{\left(2 a X+2 a^{-1} T\right)}+1}
\end{aligned}
$$

From the known three solutions $u_{0}=v_{0}=0$,

$$
\begin{aligned}
& u_{1}=4 \arctan e^{a X+a^{-1} T}, \\
& v_{1}=2 \frac{e^{\left(a X+a^{-1} T\right)}}{e^{\left(2 a X+2 a^{-1} T\right)}+1}, \\
& u_{2}=4 \arctan e^{b X+b^{-1} T}, \\
& v_{2}=2 \frac{e^{\left(b X+b^{-1} T\right)}}{e^{\left(2 b X+2 b^{-1} T\right)}+1},
\end{aligned}
$$

we can get the following new solution using the nonlinear superposition formula similarly as in [10]: 

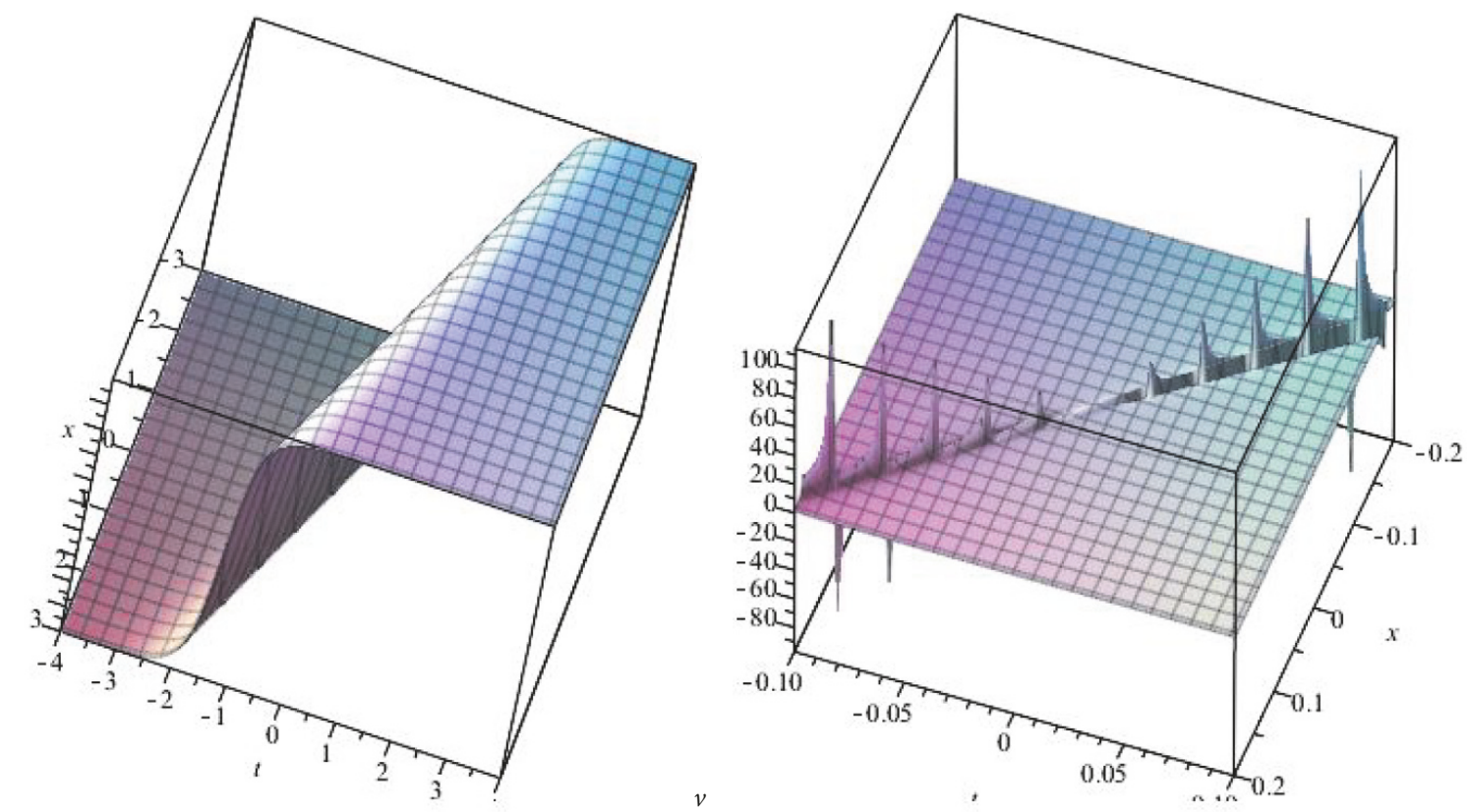

FIGURE 2: The solution $\left(u_{3}, v_{3}\right)$ of the coupled sine-Gordon equation when $a=0.5, b=1$.

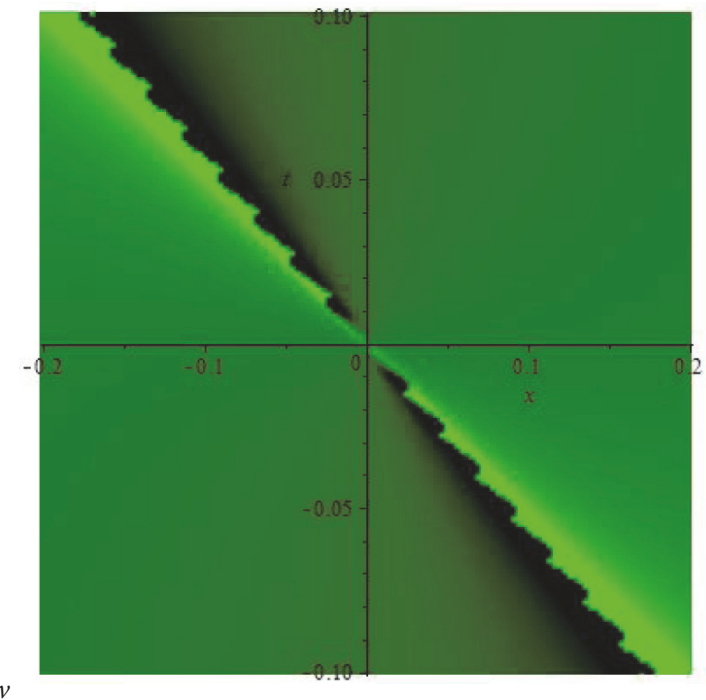

FIgURE 3: The density plot of the solution $v_{3}$ of the coupled sine-Gordon equation when $a=0.5, b=1$.

$$
\begin{aligned}
& u_{3}=4 \arctan \left(\frac{b+a}{b-a} \frac{\sinh (1 / 2)\left((a+b) X+\left(a^{-1}+b^{-1}\right) T\right)}{\cosh (1 / 2)\left((a+b) X+\left(a^{-1}+b^{-1}\right) T\right)}\right) \\
& v_{3}=\frac{a v_{1} \cos \left(u_{1} / 2\right)+a\left(v_{1}-v_{2}\right) \cos \left(\left(u_{3}-u_{2}\right) / 2\right)-b v_{2} \cos \left(u_{2} / 2\right)}{b \cos \left(\left(u_{3}-u_{1}\right) / 2\right)} .
\end{aligned}
$$

In this way, by the algebraic iterated operation we can get many new solutions of the coupled sine-Gordon equation and its inhomogeneous system.
When $X=x, T=t$, the solutions are exactly the solutions of the coupled sine-Gordon equation [10] whose graph can be seen in Figures 1 and 2 with a density plot of $v$ in Figure 3. 

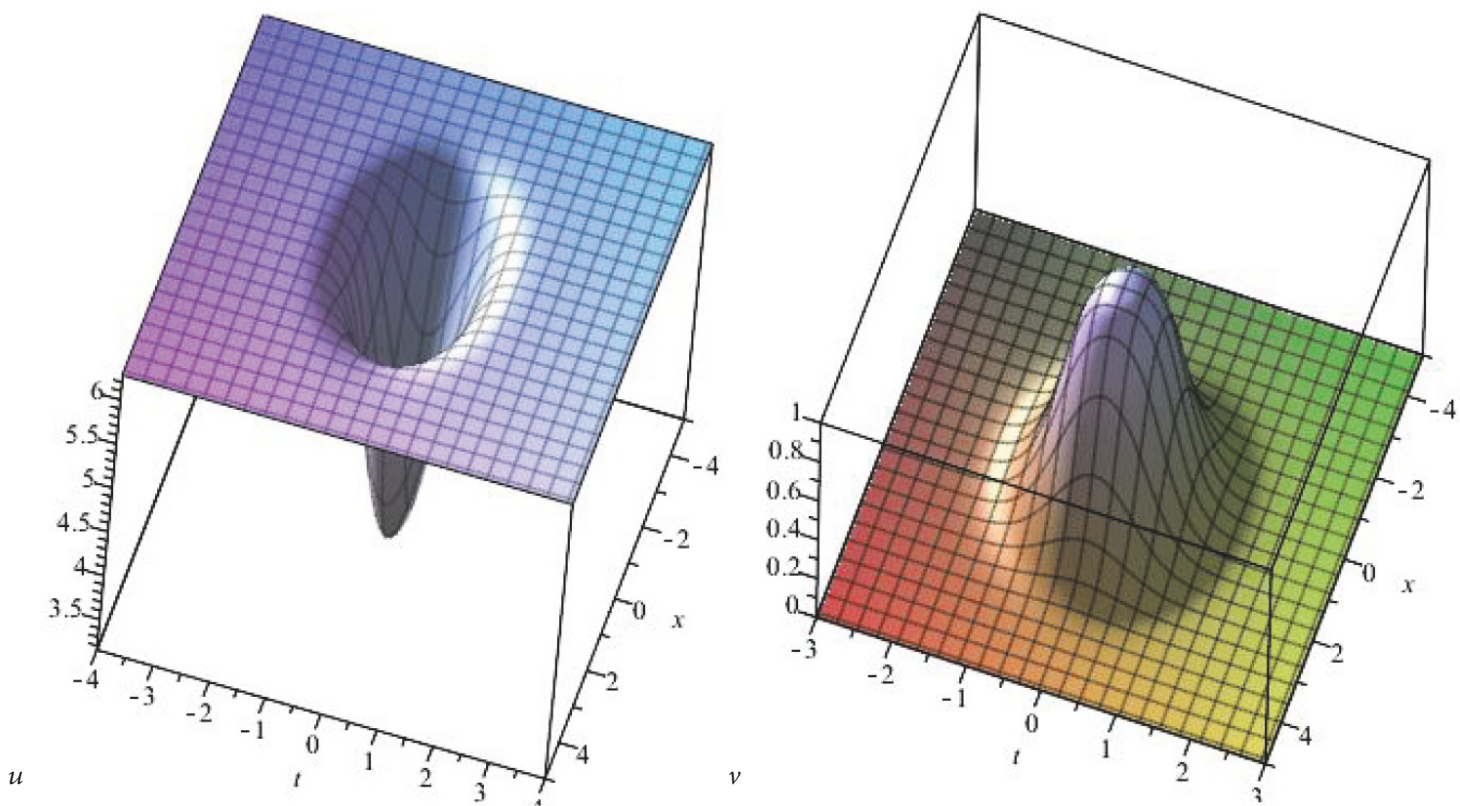

FIGURE 4: The solution $\left(u_{1}, v_{1}\right)$ of the inhomogeneous coupled sine-Gordon equation when $a=0.5, m=4 x t$.
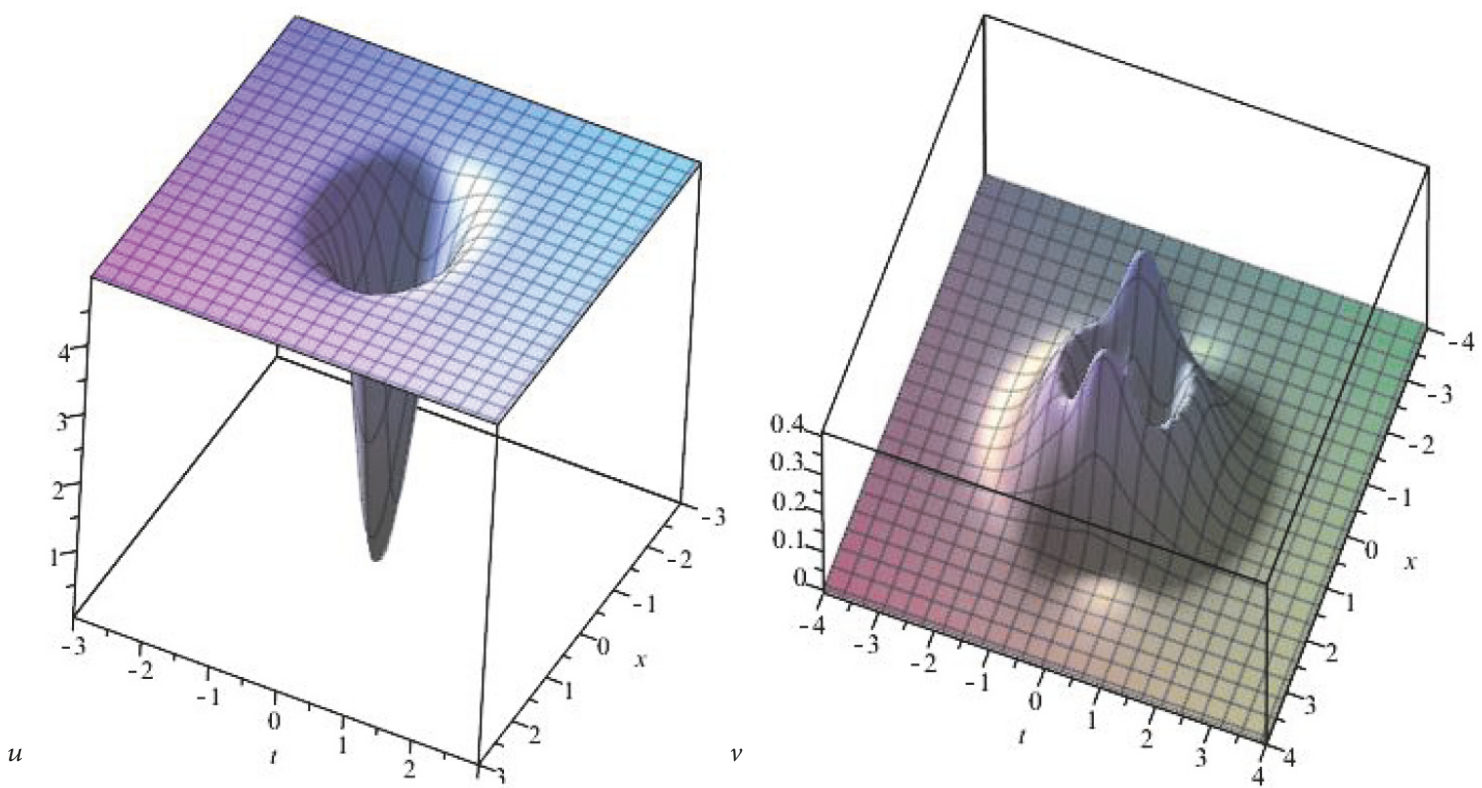

Figure 5: The solution $\left(u_{3}, v_{3}\right)$ of the inhomogeneous coupled sine-Gordon equation when $a=0.5, b=1$, and $m=4 x t$.

From these graphs, we can find that they are all traveling wave solutions such as kink and soliton solutions with a wide base pair opening configuration in DNA.

When $X=x^{2}, T=t^{2}$, the solutions of the inhomogeneous coupled sine-Gordon equation can be seen in Figures 4 and 5 with a density plot of $v$ in Figure 6.

Remark 1. The appearance of the rogue waves in the inhomogeneous coupled sine-Gordon system is due to the inhomogeneity $m(x, t)$.
As we know, the rogue waves become one of the most important topics in mathematics and physics. The rogue waves of the inhomogeneous coupled sine-Gordon system reflect highly the transient concentration of energy and the strong oscillation of spatial angles on a local part of the double helix of DNA molecules. The inhomogeneous coupled sine-Gordon system can be a good model of the interactional dynamical evolution of the base of DNA rotations in the plane perpendicular to the helical axis around 


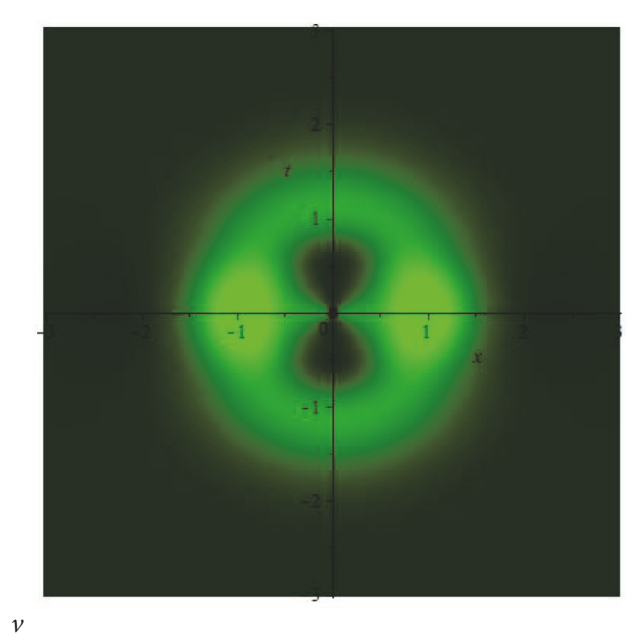

FIgURE 6: The density plot of the solution $v_{3}$ of the inhomogeneous coupled sine-Gordon equation when $a=0.5, b=1$, and $m=4 x t$.

the backbone structure in an inhomogeneous background such as ultraviolet radiations and so on.

\section{Data Availability}

No data were used to support this study.

\section{Conflicts of Interest}

The authors declare that there are no conflicts of interest regarding the publication of this paper.

\section{Acknowledgments}

This work is funded by the National Natural Science Foundation of China under Grants no. 11571192 and 81602940 and K. C. Wong Magna Fund in Ningbo University.

\section{References}

[1] M. Peyrard and A. R. Bishop, "Statistical mechanics of a nonlinear model for DNA denaturation," Physical Review Letters, vol. 62, no. 23, pp. 2755-2758, 1989.

[2] E. Goldobin, A. Sterck, T. Gaber, D. Koelle, and R. Kleiner, "Dynamics of semifluxons in $\mathrm{Nb}$ long josephson," Physical Review Letters, vol. 92, no. 5, 2004.

[3] V. G. Ivancevic and T. T. Ivancevic, "Sine-Gordon solitons, kinks and breathers as physical models of nonlinear excitations in living cellular structures," Journal of Geometry and Symmetry in Physics, vol. 31, pp. 1-56, 2013.

[4] G. A. Maugin, Nonlinear Waves in Elastic Crystals, University Press, Oxford, UK, 1999.

[5] L. V. Yakushevich, Nonlinear Physics of DNA, Wiley-VCH Verlag GmbH \& Co. KGaA, Berlin, Germany, 2004.

[6] A. Kundu, "Shape changing and accelerating solitons in the integrable variable mass sine-Gordon model," Physical Review Letters, vol. 99, no. 15, Article ID 154101, 2007.
[7] J. He, Y. Wang, and L. Li, "Non-rational rogue waves induced by inhomogeneity," Chinese Physics Letters, vol. 29, no. 6, p. 060509, 2012.

[8] C. Li and J. He, “The extended $Z_{n}$-Toda hierarchy," Theoretical and Mathematical Physics (Russian Federation), vol. 185, no. 2, pp. 1614-1635, 2015.

[9] C. Li, "Gauge transformation and symmetries of the commutative multicomponent BKP hierarchy," Journal of Physics A: Mathematical and General, vol. 49, no. 1, Article ID 015203, 2016.

[10] X. Yang and C. Li, "Bäcklund transformations of $Z_{n}$-sineGordon systems," Modern Physics Letters B. Condensed Matter Physics, Statistical Physics, Applied Physics, vol. 31, no. 17, 16 pages, 2017.

[11] M. Daniel and V. Vasumathi, "Solitonlike base pair opening in a helicoidal DNA: an analogy with a helimagnet and a cholesteric liquid crystal," Physical Review E: Statistical, Nonlinear, and Soft Matter Physics, vol. 79, no. 1, 2009. 


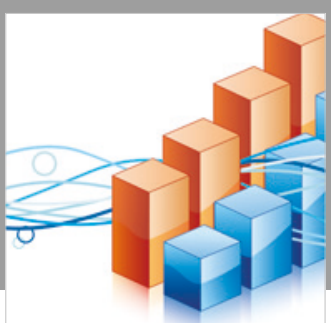

Advances in

Operations Research

\section{-n-m}
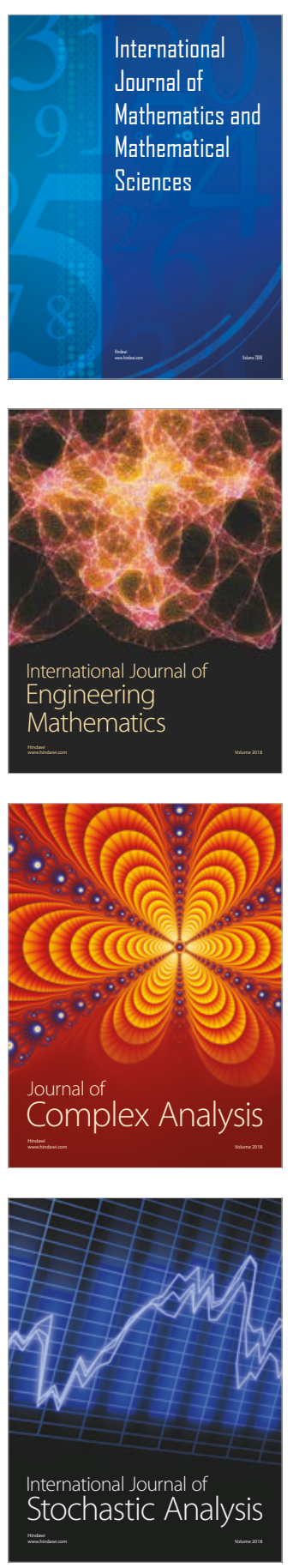
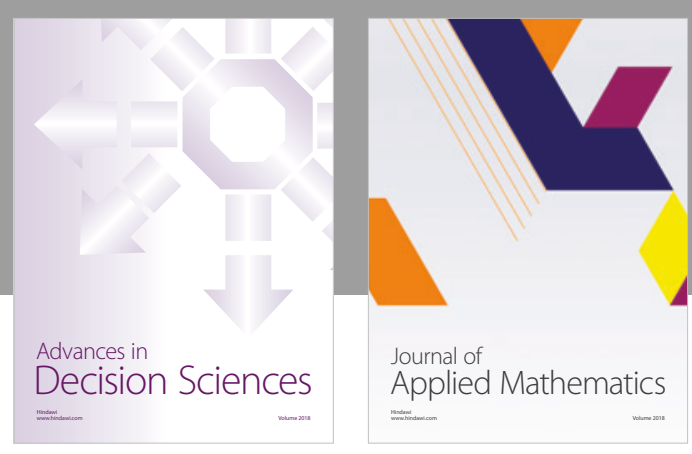

Journal of

Applied Mathematics
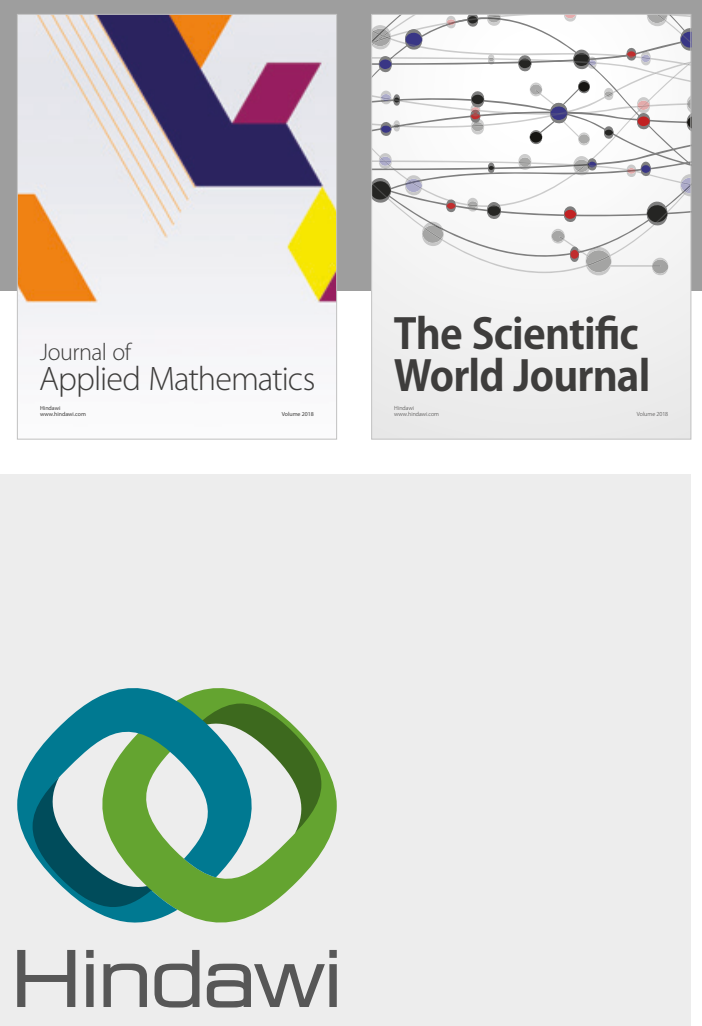

Submit your manuscripts at

www.hindawi.com

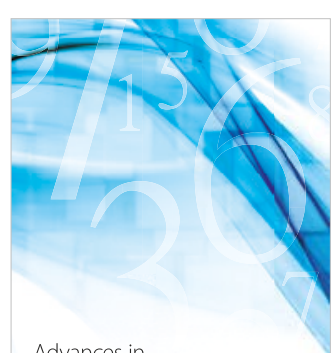

Advances in
Numerical Analysis
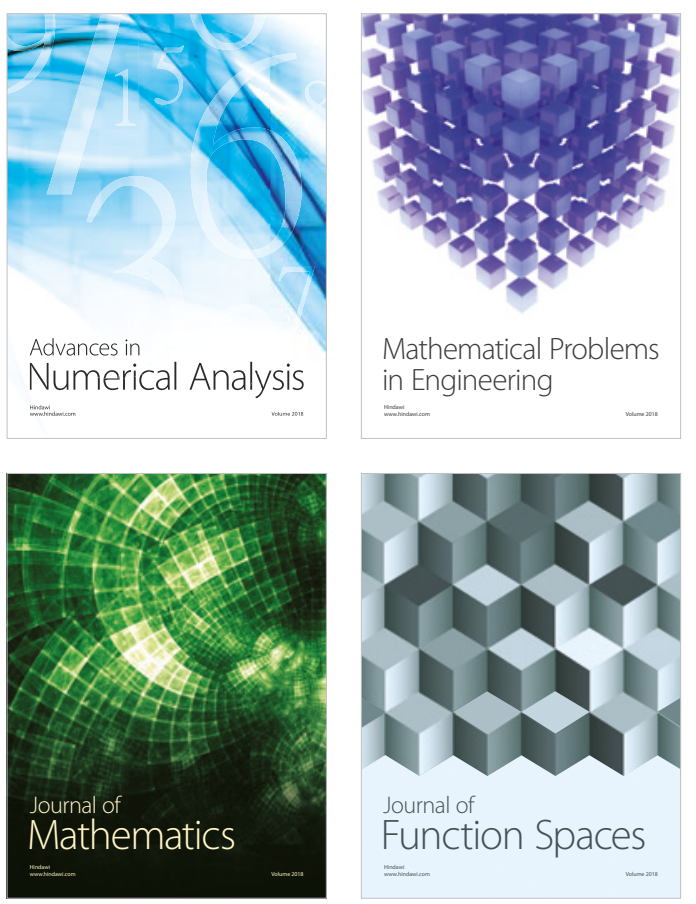

Mathematical Problems in Engineering

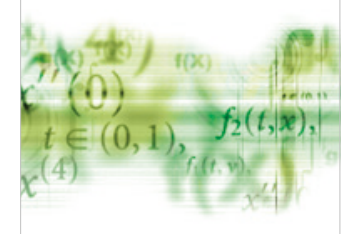

International Journal of

Differential Equations

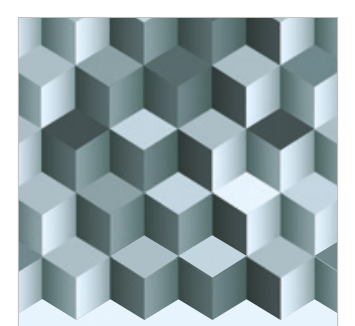

Journal of

Function Spaces

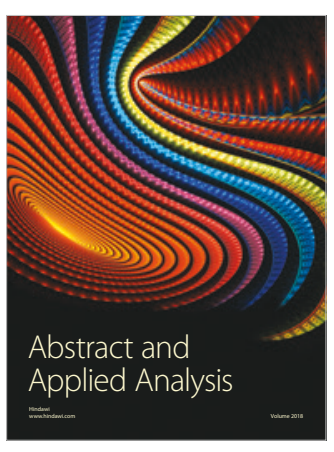

The Scientific

World Journal

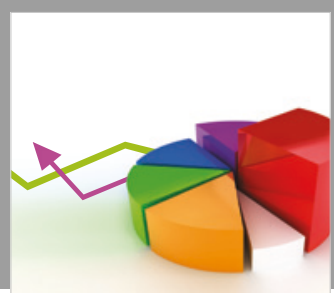

Journal of

Probability and Statistics
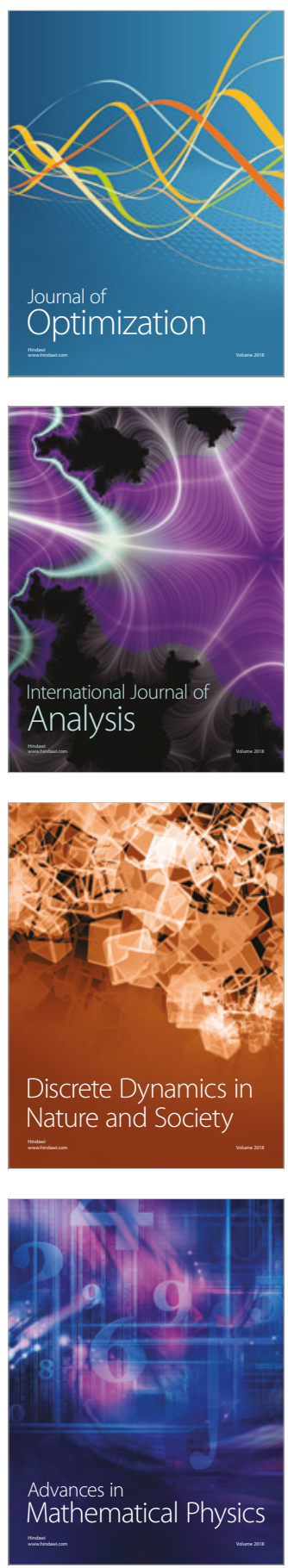